\title{
Editorial - Soziale Konflikte
}

\author{
Michael NOLLERT und Monica BUDOWSKI ${ }^{1}$
}

Universität Freiburg i. Ü.

Wer eine Gesellschaft ohne Konflikte herbeiführen will, muss dies mit Terror und Polizeigewalt tun. (Dahrendorf 1974: 275)

Liebe Leserinnen und Leser,

Gesellschaften ohne Konflikte sind zwar vorstellbar, in der empirischen Realität aber nicht zu beobachten. Und selbst autoritäre oder gar totalitäre Regimes können für sich einzig beanspruchen, dass sie in der Lage sind, mittels Überwachung und Staatsterror manifeste Konflikte (z.B. kollektive Proteste, kritische Medienberichterstattung) zu unterdrücken.

Es gehört zu den Charakteristika sowohl traditioneller als auch moderner Gesellschaften, dass ihre Mitglieder unterschiedliche Interessen und Werte haben bzw. verfolgen und diese mehr oder weniger gewaltsam aufeinanderprallen. In traditionellen hierarchisch organisierten Gesellschaften wurden Konflikte häufig durch ein Machtwort der herrschenden Kräfte beendet, auch gegen den Willen der betroffenen Mehrheit. In traditionellen akephalen Gesellschaften beruht die Konfliktbearbeitung auf einem gemeinsamen Kontext von Beziehungen und Werten und besteht aus Schlichtung, Mediation und Verhandlungen häufig unter Einbezug eines Rats der Älteren, Rats der Weisen oder von besonders respektierten Personen. In modernen demokratischen Gesellschaften werden Konflikte dagegen durch mehr oder weniger als fair eingeschätzten Verfahren bewältigt, die im Hinblick auf eine umstrittene Entscheidung, dem One man one vote-Prinzip folgend, sowohl die Interessen und kulturellen Ziele von Mehrheiten als auch von Minderheiten berücksichtigen sollten.

Von daher vermag nicht zu überraschen, dass die Frage, was soziale Konflikte sind, umstritten ist. Dem Sozialpsychologen Glasl (1999) zufolge handelt es sich bei Konflikten um eine Interaktion, bei der zwischen den beteiligten Parteien Differenzen im Wollen, Denken, Vorstellen, Interpretieren, Fühlen und Wahrnehmen zu erkennen sind. Bei soziologischen Analysen reicht es meistens aus, bei Konflikten divergente Interessen, Ziele oder Wertvorstellungen zu identifizieren. Hinzu kommt, dass in der Soziologie nicht nur Konflikte zwischen Menschen Gegenstand sind.

\footnotetext{
${ }^{1}$ Michael Nollert und Monica Budowski sind ProfessorInnen am Studienbereich Soziologie, Sozialarbeit und Sozialpolitik, Departement Sozialarbeit, Sozialpolitik und Globale Entwicklung an der Universität Freiburg (michael.nollert@unifr.ch, $\underline{\text { mo- }}$ nica.budowski@unifr.ch).
} 
Sozial sind auch Konflikte, wenn die streitenden Akteure Gruppen, Organisationen, Staaten, Allianzen von Staaten, Klassen, Ethnien oder Religionen sind. Selbst Zivilisationen (vgl. Huntington 1996) oder Generationen (vgl. Kaufmann 2005) lassen sich inzwischen als Kontrahentinnen identifizieren.

Ausserdem lassen sich in der Konfliktforschung auch Typen von Konflikten unterscheiden. Denken wir etwa an interpersonell versus intrapersonell, Intra- versus Interrollenkonflikte (vgl. Merton 1957), kalt versus heiss (Glasl 1999), symmetrisch versus asymmetrisch oder echt versus unecht (Coser 1956).

Bei der Diagnose von Konfliktursachen gehen die Meinungen auch auseinander. Während im Alltag unterschiedliche Interessen und Weltanschauungen sowie Antipathie, mangelndes Vertrauen, Betrug, Missbrauch, Aggressivität, Neid, Feindseligkeit oder Benachteiligung genannt werden, fokussiert die Soziologie auf ideologische Kontroversen, Machtgefälle, Knappheit, eine ungerechte Verteilung von Ressourcen und einen Mangel an Anerkennung (Fraser 1995), der sich neuerdings nicht mehr nur in linker, sondern auch in religiöser und rechtspopulistischer Identitätspolitik artikuliert:

What is notable, however, is how the right has adopted the language and framing of identity from the left: the idea that my particular group is being victimized, that its situation and sufferings are invisible to the rest of the society, and that the whole of the social and political structure responsible for this situation (read: the media and the political elites) needs to be smashed. (Fukuyama 2018: 122)

Wichtige Theoretiker vor der soziologischen Klassik im 19. Jahrhundert sind zweifellos Thomas Hobbes, der den autoritären Leviathan (1651) als Instrument zur Bändigung des homo lupus empfahl, sowie Adam Smith (1776), der prophezeite, dass die friedliche Konkurrenz in der Marktwirtschaft eine Win-win-Situation schaffe.

Karl Marx ist für die Konfliktsoziologie vor allem deshalb wichtig, weil er die Geschichte als Abfolge von Verteilungskonflikten zwischen ökonomischen Klassen sieht, die sich in zunehmenden ökonomischen Ungleichheiten, materieller Deprivation, häufig in gewaltsamen Auseinandersetzungen manifestieren und allenfalls in Revolutionen münden. So beginnt Kapitel 1 (Bourgeois und Proletarier) im Manifest der kommunistischen Partei mit dem berühmten Satz: „Die Geschichte aller bisherigen Gesellschaft ist die Geschichte von Klassenkämpfen“ (Marx/Engels 1972 [1848]: 462). Marx ist daher die Einsicht zu verdanken, dass viele soziale Konflikte auf Einkommens- und Vermögensungleichheiten verweisen, d.h. im Kapitalismus auf die Ausbeutung der ProletarierInnen durch die KapitalistInnen.

Max Webers Werk lässt sich sowohl als Ergänzung als auch Kritik an Marx lesen. So impliziert sein Konzept der sozialen Schliessung (vgl. Weber 2010 [1922]: 1. Teil, 1. Kapitel, \$10), dass nicht nur KapitalistInnen, sondern irgendwelche soziale Kategorien auf der Basis von askriptiven Merkmalen (Geschlecht, Hautfarbe, Religion, soziale Herkunft) und staatlicher Durchsetzung dieser Diskriminierung den Zugang zu Ressourcen blockieren können.

Frank Parkin (1979) revitalisierte Webers Konzept, wobei er an Marx monierte, dass er den Konflikten innerhalb von Klassen zu wenig Rechnung trage. Gegen Weber wandte er ein, dass er den Widerstand der Ausgeschlossenen (Usurpation) vernachlässige, z.B. die Antisklaverei-, Arbeiter-, Frauenbewegung. Zudem betonte er mit dem Konzept dual closure, dass sich in der 
Geschichte viele Beispiele doppelter Schliessung erkennen lassen (z.B. im Mittelklassenradikalismus, im Ku Klux Klan, bei Rechtspopulisten und -extremen sowie Islamisten (vgl. Ebner 2018)). Dabei wird gleichzeitig gegen oben usurpiert (z.B. Elitenkritik, Opferrhetorik) als auch gegen unten (z.B. Immigranten, Andersfarbige und -gläubige) ausgrenzt.

Im Unterschied zu Marx und Weber, die auf ökonomische Antagonismen und staatliche Protektion von privilegierten Gruppen als Ursache von Konflikten fokussieren, stehen bei Georg Simmel einerseits zwischenmenschliche Konflikte und andererseits die Konsequenzen von Konflikten im Vordergrund. So thematisierte er in seiner Studie Der Streit (1908a), wie sich die Qualität von Konflikten verändert, wenn eine Drittperson eine bilaterale Beziehung ergänzt, sei das als Kind, als Mediator, als lachender Dritter (tertius gaudens) oder teilender Herrscher (divide et impera). Von Interesse ist insbesondere auch seine Schrift Die Kreuzung sozialer Kreise (1908b, vgl. Nollert 2010), aus der sich u.a. die These ableiten lässt, dass vielfältige Beziehungsmuster von Menschen vielfältige Identitäten und damit Rollenkonflikte erzeugen, die ein tolerantes Weltbild fördern und damit die soziale Kohäsion festigen (vgl. Sen 2006). Bemerkenswert, weil äusserst aktuell, sind vor allem auch Simmels Thesen, dass Konflikte die Parteien miteinander verbinden und realer oder auch nur imaginierter Druck von aussen nicht zwangsläufig dividiert, sondern im Sinne des rally 'round the flag-Effekts selbst den Zusammenhalt von üblicherweise intern zerstrittenen Entitäten verstärkt (Coser 1956).

Obwohl zwischen Marx und Simmel aufgrund unterschiedlicher Forschungsschwerpunkte (Marx: Ursachen, Simmel: Folgen) kaum Schnittstellen erkennbar sind (vgl. Turner 1975), lassen sich bei der soziologischen Analyse historischer Phänomene beide Perspektiven durchaus verbinden. So illustrieren etwa der Imperialismus und die Weltkriege, dass die reale oder imaginierte Bedrohung von aussen den Antagonismus zwischen Kapital und Arbeit dämpfte und paradoxerweise zum Ausbau politischer Rechte (Frauenstimmrecht) und sozialer Rechte (Sozialversicherungen) beitrug (Nollert 2009).

Cosers (1956) durch Simmel inspirierte Unterscheidung zwischen echten und unechten Konflikten hilft wiederum zu verstehen, weshalb sich ökonomische Deprivation im Kapitalismus häufig nicht zwangsläufig in Widerstand gegen die ökonomischen Machtverhältnisse (echte Konflikte), sondern in Ausgrenzung kultureller Minderheiten (unechte Konflikte) artikuliert (vgl. Nollert, forthcoming).

Obwohl sich bereits die Klassiker mit Ursachen und Folgen sozialer Konflikte befassten, dominierte in der westliche Soziologie nach dem Zweiten Weltkrieg vorab die Prämisse, dass eine erfolgreiche Sozialisation und soziale Kontrolle im Hinblick auf die Konfliktbewältigung funktional sind. Mit anderen Worten: Konflikte seien dysfunktional, weil sie die Gesellschaft destabilisieren und Sozialisations- und Kontrolldefiziten aufzeigen.

Erst in den 1960er-Jahren fasste in der westlichen Soziologie wieder die Position der Klassiker Fuss, wonach Konflikte nicht a priori destruktiv wirken, sondern häufig - wie schon Marx und Simmel postulierten - progressiven sozialen Wandel vorantreiben, integrativ wirken (Coser 1956) und in Demokratien nicht zu unterdrücken, sondern zu regulieren sind (Dahrendorf 1974).

Dabei wurde die Kontroverse zwischen Funktionalismus und Konflikttheorie von Vermittlungsversuchen ergänzt. So diagnostizierte etwa Lenski (1966) eine historische Verlagerung von der funktionalen, das Überleben der Gruppe sichernden, und daher konsensuellen Verteilung 
des wirtschaftlichen Mehrwerts zur konfliktiven Verteilung von Einkommen und Vermögen in demokratischen Industriegesellschaften.

So lässt sich im 21. Jahrhundert resümieren, dass die soziologische Analyse von Konflikten nicht mehr durch die Konfliktscheu des hegemonialen Funktionalismus behindert wird. Dazu trugen im 20. Jahrhundert nicht zuletzt u.a. Norbert Elias (1977 [1939]), der Simmel-Schüler Lewis A. Coser (1956), der Liberale Ralf Dahrendorf (1957, 1974, 1994), der Weber-Schüler Frank Parkin (1979) und Randall Collins (2010) bei.

Fazit: Soziale Konflikte zwischen Menschen, Gruppen, Organisationen oder Staaten waren und sind ein Schlüsselthema der Soziologie, aber auch der konfliktregulierenden Sozialpolitik und mediationsorientierten Sozialarbeit. Im Unterschied zum funktionalistischen Gesellschaftsbild, dass die Dysfunktionalität von Konflikten betont, haben schon Marx, Weber und Simmel die konstruktiven Aspekte von Auseinandersetzungen zwischen Menschen, Gruppen oder Klassen thematisiert. In diesem Sinne teilen auch moderne Demokratien die Prämissen der Konfliktsoziologie. Kontrastierend zu vielen Epigonen von Marx, die eine Diktatur des Proletariats forderten, unterstellt die liberale Demokratietheorie indes, dass die Repression Andersdenkender destruktiv wirkt und sich der kulturelle, soziale und selbst der Klassenkonflikt mittels demokratischer Verfahren (Dahrendorf 1957, Lenski 1966, Korpi 1983) gewaltfrei bewältigen lässt.

Im Frühlingssemester 2019 fand an der Universität Freiburg die Vortragsreihe Soziale Konflikte: Typen, Ursachen und Bewältigung statt, an der sich ReferentInnen aus unterschiedlichen sozialwissenschaftlichen Disziplinen beteiligten. Das vorliegende Heft umfasst im Hauptteil vier Beiträge von ReferentInnen, die auf der Grundlage ihrer Fragestellung ihre Vorträge verschriftlicht haben.

Eddie Hartmanns Beitrag beantwortet die Frage, ob und inwiefern das soziale Medium Twitter zur gewaltsamen Eskalation der G20-Proteste in Hamburg 2017 beitrug. Ausgangspunkt ist die These, dass parallel zur Eskalation des Protestgeschehens auf den Strassen Hamburgs eine antagonistische Diskursdynamik in Twitter zu beobachten war, die eine sich verstärkenden Spirale aus Solidarisierung und Feindbildkonstruktion begünstigte. Untersucht wird die These anhand eines triangulierten Verfahrens der Netzwerk- und Inhaltsanalyse des Twitter-Diskurses. Indem die Analyse der kommunikative Praxis des Twitterns auf eine potentiell relevante soziale Mobilisierung hinweist, die mehr oder weniger gleichzeitig mit den gewaltsamen Ereignissen einhergeht, bringt der Artikel die diskursiven Dynamiken in direkten Zusammenhang mit konkreten Gewalthandlungen. Die Ergebnisse sprechen für eine Dynamik zwischen den Transformationen kognitiver Wahrnehmungs- und Deutungsmuster und ihrer polarisierenden Effekte, die wiederum gewaltsame Konflikte verursachen.

Anne Kerstens Beitrag fokussiert auf häusliche Gewalt, ein aktuelles Thema in Politik und Wissenschaft. Eine in der Wissenschaft und Öffentlichkeit kontrovers diskutierte Frage ist, ob Frauen und Männer gleichermassen von Gewalt seitens ihrer (ehemaligen) PartnerInnen betroffen sind. Ausgangspunkt des Texts ist die Kritik, dass die Forschung dazu zwar das Ausmass, mögliche Ursachen und die Folgen häuslicher Gewalt ausreichend analysierte. Die sozialpolitische Relevanz der Thematik würde jedoch vordergründig zumeist auf eine quantifizierende Perspektive abgestützt. Kersten meint, dass diese Perspektive der vielschichtigen Komplexität häuslicher Gewalt nicht gerecht werde. So argumentiert sie, dass insbesondere die 
familiären Beziehungsgefüge und -dynamiken wichtige Anhalts- und Ausgangspunkte für eine systematische und fundierte Auseinandersetzung mit dem komplexen Phänomen der häuslichen Gewalt bilden.

Amir Sheikhzadegans Beitrag befasst sich mit der Frage, weshalb sich Menschen für den Dschihadismus begeistern und folglich eine gewaltsame Verwirklichung ihrer kulturellen Ziele anstreben. Dabei geht er davon aus, dass ein adäquates Verständnis der Gründe dafür eine vertiefte Analyse der Genese dschihadistischer Identitäten voraussetzt. Von besonderer Relevanz sieht er gesellschaftliche Dynamiken, die es erlauben, ermöglichen oder fördern, dass DschihadistInnen sich neue Selbst- und Weltbilder schaffen. Die Forschung zur Genese dschihadistischer Identitäten sei indes in einem fragmentierten Zustand, womit sich eine Systematisierung identitätsrelevanter Ansätze und Studien aufdränge. Entsprechend präsentiert der Autor exemplarische Ansätze und Studien, wobei er zwischen der sozietalen Mikro-, Meso- und Makroebene sowie verschiedenen Dimensionen dieser Ebenen unterscheidet. Er gelangt zum Schluss, dass der Dschihadismus auf der Makroebene eine komplexitätsreduzierende Welttheorie darstellt, bestehend aus einem kosmischen Plan des Allmächtigen, der eine Rückkehr zum Früh-Islam vorsieht. Auf der Mikroebene würden verschiedene Prozesse und Interaktionen die Übernahme einer dschihadistischen Identität unterstützen. So liessen sich insbesondere junge Männer für den gewaltsamen Umbau der (Welt-)Gesellschaft begeistern. Dabei gebe es eine Wechselwirkung zwischen dem Handeln der DschihadistInnen und ihrer Identität, der in der Forschung besonders Rechnung getragen werden muss.

Rainer Kilbs Beitrag würdigt die Konfliktforschung als Grundlage sozialpädagogischer Gewaltprävention. Ausgehend von der konfliktsoziologischen Prämisse, dass Konflikte nicht a priori dysfunktional, sondern integrativ wirken und die gesellschaftliche Entwicklung vorantreiben, thematisiert und fordert er eine Gewaltprävention mittels offensivem Konfliktmanagement. Gefragt sind demnach konfliktfähige Bürger und Bürgerinnen, die dazu fähig sind, sich auch über den fair ausgetragenen Streit in der Vielfalt zu verständigen und selbst zu positionieren.

Ergänzt werden die vier Beiträge durch einen kürzeren Forumsbeitrag von Sarah Affentranger. Ihr Beitrag thematisiert die traditionelle jüdische Heiratsvermittlung. Dabei dokumentiert die Autorin, wie jüdische HeiratsvermittlerInnen weltweit erfolgreich versuchen, die transnationale Vergemeinschaftung des jüdischen Diaspora-Netzwerks und die Kontinuität des Judentums zu konservieren. Als translokales ethno-religiöses Netzwerk bildet die orthodoxe jüdische Diaspora somit einen Gegenpol zu modernen Jüdinnen und Juden, die nicht zuletzt auch aufgrund des Web 2.0 vermehrt ihre transkulturelle Identität akzeptieren.

Der Fokus des vorliegenden Hefts mag zwar derzeit im Schatten vielfältiger Solidaritätsakte stehen. Nach dem Ende der Corona-Krise und dem schwindenden Kohäsionsdruck (Simmel) werden aber im Hinblick auf die Frage, wer die Kosten dieser Unterstützungsleistungen tragen soll, zweifellos die alten sozialen Konfliktlinien wieder erkennbar. Spätestens dann wird die Soziologie Zeit finden, die corona-induzierten Konflikte zu analysieren, sei dies auf der Ebene zwischen Staaten, Organisationen, Gruppen oder innerhalb von dyadischen Beziehungen und in der persönlichen Entwicklung.

Wir wünschen Ihnen eine spannende und anregende Lektüre! 


\section{Literatur}

Collins, Randall (2010). Konflikttheorie: ausgewählte Schriften. Wiesbaden: Springer VS.

Coser, Lewis A. (1956). The Functions of Social Conflict. Glencoe, IL: Free Press.

Dahrendorf, Ralf (1957). Soziale Klassen und Klassenkonflikt in der industriellen Gesellschaft. Stuttgart: Ferdinand Enke.

Dahrendorf, Ralf (1974). Pfade aus Utopia. Arbeiten zur Theorie und Methode der Soziologie. 3. Auflage. München: Piper \& Co. Verlag.

Dahrendorf, Ralf (1994). Der moderne soziale Konflikt. Essay zur Politik und Freiheit. München: dtv.

Elias, Norbert (1977 [1939]). Über den Prozess der Zivilisation. Bde. 1, 2, (4. Aufl.). Frankfurt am Main: Suhrkamp.

Fraser, Nancy (1995). From Redistribution to Recognition? Dilemmas of Justice in a 'Post-Socialist Age', New Left Review I(212), 68-93.

Fukuyama, Francis (2018). Identity. Contemporary Identity Politics and the Struggle for Recognition. London: Profile Books.

Ebner, Julia (2018). Wut. Was Islamisten und Rechtsextreme mit uns machen. Darmstadt: Theiss Verlag.

Elias, Norbert (1969 [1939]). Über den Prozeß der Zivilisation. Soziogenetische und psychogenetische Untersuchungen. Bern, München: Francke.

Glasl, Fritz (1999). Konfliktmanagement. Bern: Verlag Karl Haupt.

Hobbes, Thomas (2008 [1651]) Leviathan, oder Stoff, Form und Gewalt eines kirchlichen und bürgerlichen Staates. Oldenburg: Akademie Verlag.

Huntington, Samuel P. (1996). The Clash of Civilizations and the Remaking of World Order. New York: Simon \& Schuster.

Kaufmann, Franz-Xaver (2005). Schrumpfende Gesellschaft. Vom Bevölkerungsrückgang und seinen Folgen, Frankfurt am Main: Suhrkamp.

Korpi, Walter (1983). The Democratic Class Struggle. London: Routledge \& Kegan Paul.

Lenski, Gerhard (1966). Power and Privilege: A Theory of Social Stratification. New York: McGraw-Hill.

Marx, Karl und Friedrich Engels (1972 [1848]). Manifest der kommunistischen Partei, in: dies. Werke, Band 4, 6. Berlin: Dietz Verlag.

Merton, Robert K. (1957). The Role-Set. Problems in Sociological Theory. British Journal of Sociology 8(2), 106-120.

Nollert, Michael (2009). Der Krieg als Vater von Demokratie und Sozialstaat? Der Militarisierungsschub nach der Französischen Revolution und die Expansion politischer und sozialer Rechte, In: Maeder, Christoph, Ueli Mäder und Sarah Schilliger (Hg.). Krieg. Zürich: Seismo. 184-206.

Nollert, Michael (2010). Kreuzung sozialer Kreise. Auswirkungen und Wirkungsgeschichte. In: Stegbauer, Christian und Roger Häußling (Hg.). Handbuch Netzwerkforschung. Wiesbaden: VS Verlag für Sozialwissenschaften, 159-167. 
Nollert, Michael (forthcoming). External threats and multiple identities as basis of social cohesion: Simmel's legacy in the analysis of social conflicts. In: Pedro Caetano (Ed.) Simmel and Beyond. Dordrecht: SpringerOpen.

Parkin, Frank (1979). Marxism and Class Theory: A Bourgeois Critique. New York: Columbia University Press.

Sen, Amartya (2006). Identity and Violence. The Illusion of Destiny. London: Allen Lane.

Simmel, Georg (1908a). Der Streit. In: ders. Soziologie. Untersuchungen über die Formen der Vergesellschaftung. Berlin: Duncker \& Humblot, 186-255.

Simmel, Georg (1908b). Die Kreuzung sozialer Kreise. In: ders. Soziologie. Untersuchungen über die Formen der Vergesellschaftung. Berlin: Duncker \& Humblot, 305-344.

Smith Adam (2005 [1776]). Untersuchung über Wesen und Ursachen des Reichtums der Völker. Tübingen: UTB.

Turner, Jonathan H. (1975). Marx and Simmel revisited: Reassessing the foundations of conflict theory. Social Forces, 53(4), 618-627.

Weber, Max (2010 [1922]). Wirtschaft und Gesellschaft. Frankfurt am Main: Zweitausendeins-Verlag. 